\title{
SEGURANÇA DO PACIENTE: CONHECIMENTO DE ALUNOS DE GRADUAÇÃO EM ENFERMAGEM
}

Denise Franze Bogarin ${ }^{1}$, Ariane Cristina Barboza Zanetti ${ }^{2}$, Maria de Fátima Paiva Brito ${ }^{3}$, Juliana Pereira Machado ${ }^{4}$, Carmen Silvia Gabriel ${ }^{5}$, Andrea Bernardes ${ }^{5}$

'Enfermeira. Universidade de São Paulo. Ribeirão Preto-SP-Brasil.

${ }^{2}$ Farmacêutica. Mestre em Ciências. Universidade de São Paulo. Ribeirão Preto-SP-Brasil.

${ }^{3}$ Enfermeira. Mestre em Enfermagem. Universidade de São Paulo. Ribeirão Preto-SP-Brasil.

${ }^{4}$ Enfermeira Especialista em Gestão em Saúde. Universidade de São Paulo. Ribeirão Preto-SP-Brasil.

${ }^{5}$ Enfermeira. Doutora em Enfermagem. Universidade de São Paulo. Ribeirão Preto-SP-Brasil.

RESUMO: Estudo com o objetivo identificar o conhecimento de alunos em Enfermagem de uma universidade pública do interior paulista sobre segurança do paciente. Trata-se de estudo quantitativo, transversal, entre maio e junho de 2011. Participaram 118 alunos regularmente matriculados no terceiro, quarto e quinto anos. Questionário autoaplicável foi analisado com estatística descritiva. Os resultados apontam que parte dos alunos considera que a assistência de enfermagem é insegura e pode trazer riscos. A falta de preparo da equipe é considerada como fator dificultador para que se preste assistência segura. Além disso, grande parte dos alunos desconhece o termo evento adverso. Conclui-se que os alunos conseguem contemplar os aspectos relacionados à segurança do paciente e as responsabilidades do enfermeiro para alcance de assistência segura. Porém, é necessário ampliar o escopo do ensino sobre esta temática.

DESCRITORES: Serviço hospitalar de Enfermagem; Medidas de segurança; Segurança do paciente.

\section{PATIENT SAFETY: KNOWLEDGE OF UNDERGRADUATE NURSING STUDENTS}

\begin{abstract}
Study to identify the knowledge of Nursing students at a public university in the interior of the State of São Paulo about patient safety. Quantitative, crosssectional study developed between May and June 2011. The participants were 118 students regularly enrolled in the third, fourth and fifth year. A self-applied questionnaire was analyzed with the help of descriptive statistics. The results indicate that part of the students consider that nursing care is unsafe and can entail risks. The team's lack of preparation is considered a factor that hampers safe care delivery. In addition, a large part of the students is unfamiliar with the term adverse event. In conclusion, the students are able to consider aspects of patient safety and nurses' responsibilities to achieve safe care. Nevertheless, the scope of teaching on this theme needs to be expanded. DESCRIPTORS: Nursing service, Hospital; Security measures; Patient safety.
\end{abstract}

\section{SEGURIDAD DEL PACIENTE: CONOCIMIENTO DE ALUMNOS DE GRADUACIÓN EN ENFERMERÍA}

RESUMEN: Estudio con el objetivo de identificar el conocimiento de alumnos en Enfermería de una universidad pública del interior de São Paulo sobre seguridad del paciente. Es un estudio cuantitativo, transversal, realizado entre mayo y junio de 2011. Participaron 118 alumnos matriculados de modo regular en el tercero, cuarto y quinto años. Cuestionario autoaplicable fue analizado con estadística descriptiva. Los resultados apuntan que parte de los alumnos considera que la asistencia de enfermería presenta inseguridad y puede traer riesgos. La falta de preparación del equipo es considerada factor dificultador para que se presente una asistencia segura. Además de eso, gran parte de los alumnos desconoce el término evento adverso. Se concluye que los alumnos logran contemplar los aspectos relacionados a la seguridad del paciente y las responsabilidades del enfermero para el alcance de la asistencia segura. Sin embargo, se necesita ampliar el escopo de la enseñanza acerca de esta temática.

DESCRIPTORES: Servicio hospitalar de Enfermería; Medidas de seguridad; Seguridad del paciente. 


\section{INTRODUÇÃO}

No ano 2004 foi criada a Aliança Mundial para Segurança do Paciente pela Organização Mundial de Saúde. Tal Aliança foi concebida com o objetivo de despertar a consciência e o comprometimento político para melhorar a segurança na assistência, além de apoiar os países no desenvolvimento de políticas públicas e práticas para segurança do paciente em todo o mundo ${ }^{(1)}$.

Segurança pode ser definida como ausência de exposição ao perigo e proteção contra ocorrência ou risco de lesão ou perda ${ }^{(2)}$. Para a Organização Mundial da Saúde, segurança do paciente consiste na ausência de dano potencial ou desnecessário para o paciente associado aos cuidados em saúde ${ }^{(3)}$.

A segurança do paciente é um tema atual e de importância acentuada. Dados da literatura indicam que, na Europa, há mais de 10 anos, a cada dez pacientes hospitalizados sofriam danos e eventos adversos evitáveis, ocasionados durante a assistência recebida ${ }^{(4)}$. Outra informação complementar, porém bastante recente, mostra que cerca de 50 a $60 \%$ destes eventos são evitáveis ${ }^{(5-6)}$.

Por acompanhar toda a trajetória do paciente durante sua permanência no hospital e ser o responsável por coordenar a assistência de enfermagem prestada, o enfermeiro tem papel fundamental na promoção da segurança do paciente durante o processo assistencial( ${ }^{(7)}$. Isso evoca a necessidade de capacitação científica desse profissional, comprometendo-se eticamente com ações sistêmicas de avaliação e prevenção, e tentando viabilizar a redução de desfechos indesejados, bem como analisar o impacto sobre a qualidade do cuidado oferecido ${ }^{(8)}$.

Em qualquer análise de qualidade, a falta de informações sobre os eventos adversos com os pacientes e seus fatores causais são problemáticas graves com que os gestores e pesquisadores se deparam, impedindo o conhecimento, avaliação e discussão sobre as consequências destes eventos para os profissionais, usuários e familiares. Esta lacuna prejudica a ação dos gestores no planejamento e desenvolvimento de estratégias voltadas para a adoção de práticas seguras, minimização dos eventos e melhoria da assistência, colocando em risco a segurança dos pacientes $^{(9)}$.

A complexidade do cuidado à saúde enfrentada pelos enfermeiros nos últimos tempos tem proporcionado discussões acerca de se investir na geração de futuros profissionais mais críticos e reflexivos para proporcionar um cuidado mais seguro. Consequentemente, torna-se imprescindível aos acadêmicos de enfermagem, como novatos na profissão, que a segurança do paciente seja agregada ao ensino como um valor essencial de enfermagem humanística e direito vital do paciente ${ }^{(10)}$.

Para que ocorra mudança da cultura de segurança nas instituições de saúde, os novos profissionais devem apresentar conhecimentos e habilidades para identificar e tomar medidas cabíveis após a ocorrência de um erro. Cursos de graduação da área da saúde podem desempenhar importante papel na promoção de conceitos e habilidades a respeito do erro humano e segurança do paciente. Estudos demonstram que, ao serem inseridos nessa temática, os alunos são encorajados e reconhecem a relevância desse conteúdo para sua formação, além de ser possível distinguir o grande impacto na assistência prestada ao paciente $\mathrm{e}^{(11-13)}$.

Frente ao exposto, este trabalho tem como objetivo identificar o conhecimento de alunos do curso de bacharelado e licenciatura em enfermagem de uma universidade pública do interior paulista sobre segurança do paciente, podendo interessar a um amplo público que também ambicione investigar aspectos pautados pela segurança do paciente, especificamente no que se refere aos graduandos dos cursos de enfermagem distribuídos por todo o país, uma vez que a mesma estratégia pode ser aplicada a diferentes instituições de ensino. Ainda, o assunto tratado apresenta um enfoque contemporâneo, principalmente devido à recente publicação dos Protocolos Básicos de Segurança do Paciente do Programa Nacional de Segurança do Paciente, aprovados pelas Portarias n. 1.377/2013 ${ }^{(14)} \mathrm{e}$ 2.095/2013(15) do Ministério da Saúde.

\section{MÉTODO}

Trata-se de um estudo transversal, descritivoexploratório ${ }^{(16)}$, com abordagem quantitativa, 
realizado em uma universidade pública do interior do Estado de São Paulo, entre maio e junho de 2011. Participaram como população do estudo alunos regularmente matriculados nos cursos de licenciatura e bacharelado em enfermagem do terceiro, quarto e quinto anos. A pesquisa foi aprovada pelo Comitê de Ética em Pesquisada da Escola de Enfermagem de Ribeirão Preto da Universidade de São Paulo (protocolo n. 1149/2010) e considerou todos os princípios éticos para o cumprimento das normas em pesquisas científicas envolvendo seres humanos.

Para coleta de dados utilizou-se um questionário semiestruturado, autoaplicável, composto por seis questões fechadas e quatro questões abertas, abordando a temática referente à segurança do paciente e à qualidade da assistência de enfermagem e baseado na literatura, previamente testado em um grupo equivalente de 10 alunos, excluídos da amostra final. Antes da coleta, os pesquisadores esclareceram todas as dúvidas, pretendendo facilitar o entendimento sem comprometer as respostas dos alunos.

Os resultados das questões fechadas foram analisados em frequência e porcentagem utilizando o sistema Epi Infoß. Já os resultados das questões abertas foram analisados de forma descritiva, através da análise e classificação do conteúdo das respostas.

\section{RESULTADOS}

Para identificar o conhecimento de alunos do curso de licenciatura em enfermagem sobre segurança do paciente, este estudo obteve respostas de 118 alunos, sendo do terceiro (38), quarto (39) e quinto ano (41), com idade entre 19 e 35 anos. A maioria (84,7\%) dos entrevistados era do sexo feminino.
Sobre a definição de segurança do paciente na assistência de enfermagem hospitalar, o conteúdo das respostas dos alunos do terceiro ano destacou que são os cuidados prestados ao paciente garantindo uma melhor recuperação, boa higiene, biossegurança, não expondo o paciente a outras doenças, além das responsabilidades no desempenho dos procedimentos, sem prejuízos à saúde.

A análise das respostas dos alunos do quarto ano demonstrou que, para eles, segurança é evitar que o estado de saúde do paciente se agrave, enquanto que, para os alunos do terceiro ano, segurança é realizar todos os procedimentos necessários de maneira segura, responsável, baseando-se em manter a saúde do paciente, evitar iatrogenias e promover o bem-estar com uma assistência adequada.

Segundo os alunos do quinto ano, segurança é a realização de procedimentos que visem à ausência de danos para o paciente, prevenção de fatores de risco, minimização de danos físicos e psicológicos/promoção de cuidado, proteção do paciente, promoção de integridade, ambiente e técnicas seguros e atendimento humanizado.

Quando questionados sobre a possibilidade da assistência de enfermagem trazer riscos aos pacientes, 94,7\% dos alunos do terceiro ano, $92,3 \%$ do quarto ano e $95,1 \%$ do quinto ano assentiram positivamente. Verificou-se ainda que, $52,6 \%$ dos alunos do terceiro ano, 35,9\% do quarto ano e $48,8 \%$ do quinto ano responderam que a assistência de enfermagem oferecida não é segura para o paciente.

Analisou-se também nesta pesquisa quais aspectos são considerados fatores que dificultam ou impedem que a enfermagem desenvolva uma assistência com menores riscos ao paciente no âmbito hospitalar, e as respostas encontram-se distribuídas na Tabela 1.

Tabela 1 - Dificuldades impostas para a enfermagem desenvolver uma assistência com menores riscos ao paciente no âmbito hospitalar, segundo alunos de licenciatura em Enfermagem. Ribeirão Preto-SP-Brasil, 2011

\begin{tabular}{lccc}
\hline Dificuldades apontadas & $\mathbf{3}^{\circ}$ ano (\%) & $\mathbf{4}^{\circ}$ ano (\%) & $\mathbf{5}^{\circ}$ ano $(\mathbf{\%})$ \\
\hline Conhecimento da equipe acerca do tema & 71,1 & 64,1 & 73,2 \\
\hline Falhas na estrutura física das unidades & 78,9 & 89,7 & 75,6 \\
\hline Dimensionamento da equipe & 63,2 & 66,7 & 78,0 \\
\hline Disponibilidade e qualidade de materiais e medicamentos & 63,2 & 84,6 & 73,2 \\
\hline Preparo técnico - teórico da equipe & 89,5 & 92,3 & 80,5 \\
\hline $\begin{array}{l}\text { Políticas gerenciais dos hospitais e serviços de enfermagem relacionada } \\
\text { à ocorrência de eventos adversos }\end{array}$ & 28,9 & 66,7 & 61,0 \\
\hline
\end{tabular}


No que se refere ao conhecimento do termo "eventos adversos", os dados apontam que $47,4 \%$ dos alunos do terceiro ano, 69,2\% do quarto ano e $46,3 \%$ do quinto ano não sabem o significado do termo. Dentre os que referem conhecer o termo, descrevem-no como algo que não sai conforme previsto; um evento indesejado ou algo que foge ao esperado.

Para os alunos do terceiro ano são erros de medicamentos e procedimentos; queda de pacientes e infecção hospitalar. Já para os alunos do quarto ano são fatos que ocorrem por despreparo da equipe, falta de conhecimento ou uma intercorrência; riscos não programados; algo que prejudique/interfira no curso da assistência; um evento que contradiz o esperado, como falhas no procedimento. Por fim, para os alunos do quinto ano são eventos que fogem ao controle da equipe; um erro cometido pelo profissional; um evento indesejado ou que resulte em dano ao paciente; uma complicação ocorrida por ação realizada inadequadamente.

Em relação aos eventos adversos conhecidos pelos alunos, o Quadro 1 sintetiza as respostas.

Quadro 1 - Eventos adversos relacionados à assistência de enfermagem, segundo alunos de licenciatura em Enfermagem. Ribeirão Preto-SP-Brasil, 2011

\begin{tabular}{|l|c|c|c|}
\hline EVENTO ADVERSO & $\mathbf{3}^{\circ}$ ano & $\mathbf{4}^{\mathbf{0}}$ ano & $\mathbf{5}^{\mathbf{0}}$ ano \\
\hline Queda & $\mathrm{x}$ & $\mathrm{x}$ & $\mathrm{x}$ \\
\hline Erro de medicação & $\mathrm{x}$ & $\mathrm{x}$ & $\mathrm{x}$ \\
\hline Infecção hospitalar & $\mathrm{x}$ & $\mathrm{x}$ & $\mathrm{x}$ \\
\hline Erro /falha nos procedimentos & $\mathrm{x}$ & & $\mathrm{x}$ \\
\hline Contaminação de feridas & & $\mathrm{x}$ & $\mathrm{x}$ \\
\hline UPP (Úlceras de pressão) & & $\mathrm{x}$ & $\mathrm{x}$ \\
\hline Flebites & & $\mathrm{x}$ & $\mathrm{x}$ \\
\hline Uso de materiais de má qualidade/inadequados & & & $\mathrm{x}$ \\
\hline Contaminação cruzada & & & $\mathrm{x}$ \\
\hline Falha na identificação do paciente & & & $\mathrm{x}$ \\
\hline Prescrições erradas & & & $\mathrm{x}$ \\
\hline Descarte de material de forma imprópria, & & & \\
\hline Acidentes de trabalho & & & \\
\hline
\end{tabular}

\section{DISCUSSÃO}

Os resultados demonstram que os alunos possuem entendimento essencial referente à temática, reconhecem estratégias e sabem da sua importância na assistência. De tal modo, percebe-se que todos os participantes, de maneira uniforme, possuem compreensão do significado de segurança do paciente e reconhecem a necessidade de melhorias nesse aspecto nos serviços hospitalares.

Falhas nas estruturas físicas das unidades foram alguns dos aspectos mais ressaltados pelos alunos como fator para se prestar uma assistência com menores riscos, concordando com a literatura, a qual cita que uma boa infraestrutura promove a qualidade e a produtividade. Entretanto, a excelência da planta física e dos equipamentos apenas informa o potencial da instituição, e não avalia a sua efetividade e eficiência. Neste sentido, o elemento humano que presta a assistência, com seus valores morais, formação profissional, atualização técnico-científica e comprometimento, influencia muito mais na qualidade da assistência e produtividade ${ }^{(17)}$.

Em relação ao preparo teórico-prático da equipe, estratégia citada pelos alunos e que segundo eles, consta em disciplinas de graduação e cenário de prática hospitalar, um estudo nacional ${ }^{(18)}$ afirma que a equipe de enfermagem necessita não somente de conhecimentotécnico do procedimento que irá realizar, mas também do referencial teórico para tal. Portanto, o trabalho será realizado de maneira qualificada se houver uma vinculação entre teoria e prática.

Os resultados versam assertivamente sobre a importância do dimensionamento na segurança do paciente. De acordo com a literatura, o dimensionamento da equipe de enfermagem deve incluir todas as categorias e utilizar metodologias e critérios que permitam adequação 
dos recursos humanos em termos quantitativos e qualitativos. Dimensionar a quantidade de trabalhadores necessários à assistência de enfermagem implica na identificação e caracterização da clientela, estruturando a equipe com o anseio de atender as demandas de cuidados e melhorar a qualidade da assistência ${ }^{(19)}$.

Outro aspecto corretamente citado foi o dimensionamento e qualidade de materiais e medicamentos, pois é difícil disseminar a segurança do paciente quando os recursos materiais são escassos ou o suporte tecnológico é deficiente, acrescido de uma política economicista que oculta erros e de uma dispersão de recursos entre as instituições privadas e estatais ${ }^{(20)}$.

Apesar de grande parte dos alunos ter declarado desconhecer o termo evento adverso, observou-se coerência entre as definições citadas pelos alunos e as encontradas na literatura, na qual se verifica que evento adverso é todo evento que ocorre durante a assistência à saúde e que pode causar uma injúria, dano físico ou psicológico ao paciente ou à organização ${ }^{(21)}$. Adicionalmente, uma pesquisa brasileira considerou os eventos relacionados aos indicadores de qualidade, medicação, quedas, cateteres, sondas, drenos e à integridade da pele são mais frequentes ${ }^{(22)}$. Tais asseverações são semelhantes às respostas provenientes dos alunos deste estudo.

Em geral, o erro de medicação foi o evento adverso mais citado pelos alunos. Entre os fatores que podem originar tais erros se destacam a caligrafia ilegível ou ambiguidade na prescrição médica, separação de medicamentos distintos dos prescritos, erro na via de administração e interação medicamentosa ${ }^{(23)}$.

Neste contexto, uma das estratégias referidas pelos alunos, faz menção à prescrição médica eletrônica, a qual constitui uma das maneiras de prevenção e redução de erros de medicação, pois facilita a leitura dos dados, é mais vantajosa, organizada, rápida e prática para o manuseio dos profissionais ${ }^{(24)}$.

Outra estratégia devidamente mencionada é a identificação dos pacientes nas instituições de saúde, que também constitui um problema potencial na cadeia de análise dos eventos adversos relacionados à administração de medicamentos e, portanto, estudos mais sistematizados acerca da viabilidade do sistema de identificação adotado nas instituições precisariam ser realizados ${ }^{(17,25)}$.
Pesquisas têm demonstrado que algumas mudanças simples em práticas de assistência são capazes de reduzir os eventos adversos. Exemplos disso são as ações que incentivam a lavagem das mãos, diminuindo as taxas de infecções nos ambientes de cuidado ao paciente ${ }^{(25)}$, iniciativa aludida pela maioria dos alunos neste estudo, como estratégia abordada em disciplina do curso de enfermagem e observada no âmbito da prática hospitalar. Outra tática importante e que favorece a segurança do paciente é o gerenciamento de riscos, consistindo em identificar a provável origem dos eventos adversos, avaliar os danos causados e tomar as decisões apropriadas concernentes a esses problemas ${ }^{(26)}$.

Em relação à comunicação, essa é uma importante ferramenta abordada na graduação, porém não observada na prática hospitalar. Há evidências científicas de que as relações interpessoais se encontram prejudicadas nas instituições de saúde, uma vez que são poucas as ocasiões em que é possível deparar-se com uma relação entre paciente e profissional e, quando isso ocorre, as experiências positivas são suprimidas frente à forte expressão dos múltiplos eventos negativos ${ }^{(27)}$.

Ressalta-se que muitas táticas de melhoria na segurança do paciente mencionadas pelos alunos foram abordadas em disciplinas durante a graduação, mas não foram observadas nos cenários de prática hospitalar. Esta informação corrobora com trabalhos científicos que afirmam que há uma pendência entre o que é ensinado e o que realmente acontece nos cenários de prática dos profissionais da saúde, de modo que a enfermagem necessita transformar o discurso da pesquisa sobre segurança do paciente em uma atuação sólida ${ }^{(9,27)}$.

Contudo, sabe-se que os currículos dos cursos de enfermagem não possuem uma estratégia referente à segurança do paciente. Não existem regulamentos de inserção de cursos específicos em segurança ou determinado número de horas para o ensino da temática em sala de aula, laboratório ou clínica. Também não há qualquer base de dados dedicada a informar a abrangência das ações concernentes à segurança do paciente nos programas de bacharelado ou licenciatura. Por isso, recentemente, inserções curriculares a respeito da segurança do paciente no âmbito clínico vêm surgindo na literatura e, além disso, 
educadores e outros interessados podem, de forma autônoma, planejar, implementar e avaliar algumas iniciativas em segurança do paciente ${ }^{(10)}$.

Nesse contexto, ambientes hospitalares que prezam pela segurança de seus pacientes são aqueles nos quais há avaliações constantes, os profissionais são educados, cria-se uma cultura não punitiva, incentiva-se o relato de erros e se simplifica os processos, tudo isso com o escopo de interceptar os possíveis erros e falhas antes que eles atinjam o paciente ${ }^{(28)}$. Para tanto, as organizações de saúde devem possuir controle de todos os seus processos críticos, sobretudo no conhecimento da complexidade dos fatores de riscos envolvidos nos procedimentos ${ }^{(29)}$.

O estudo realizado apresentou uma limitação importante no que se refere ao tamanho da amostra, que se apresenta em número reduzido, permitindo considerar que os resultados encontrados se aplicam somente a população em questão. Assim sendo, pelo fato da amostra deste trabalho englobar estudantes de uma única instituição de ensino superior, não é possível generalizar os resultados obtidos para estudantes oriundos de outras instituições.

\section{CONSIDERAÇÕES FINAIS}

Esse estudo versou sobre o conhecimento dos alunos de graduação em enfermagem sobre segurança do paciente e seus resultados trazem evidências sobre o entendimento de conceitos e estratégias voltadas a essa segurança na assistência hospitalar.

Concluiu-se que os alunos conseguem contemplar vários aspectos relacionados à segurança do paciente; as responsabilidades do enfermeiro para alcance de assistência segura, e reconhecem estratégias para disseminá-la. Também, compreendem que a assistência pode trazer riscos e não é segura, havendo variações dentre os alunos de anos diferentes.

Vale destacar que, para os alunos o alcance do cuidado seguro se dá principalmente por meio de aptidão teórico-prática derivada da equipe de saúde, não se evidenciando nos resultados que eles entendam os aspectos dos processos multiprofissionais e de política institucional como fatores decisivos para melhoria da segurança assistencial.
É imprescindível que os cursos de graduação em enfermagem abordem a segurança do paciente durante o processo de formação, subsidiando ações de enfermagem e estabelecendo conhecimentos e habilidades acerca de estratégias que minimizem erros e cultivem a segurança do paciente, que não estejam apenas voltadas para questões de conhecimento técnico, mas sim da necessidade de se trabalhar a segurança enquanto cultura organizacional e ferramenta gerencial para $\mathrm{o}$ enfermeiro.

Por fim, quanto às contribuições deste estudo para a sociedade, almeja-se munir os enfermeiros com conhecimentos sobre segurança do paciente, competências e atitudes, a fim de aprimorar a qualidade e a segurança do cuidado em saúde no país e despertar o interesse de educadores e educandos para esta questão. Em síntese, o desenvolvimento de competências indicativas da qualidade e segurança da assistência, durante a graduação em enfermagem, deve ser considerado como prática essencial para a formação de um profissional comprometido com a segurança do paciente.

\section{REFERÊNCIAS}

1. Joint Commission for Patient Safety. World Alliance for Patient Safety [Internet]. 2008. [acesso em 01 ago 2013]. Disponível: http://www.ccforpatientsafety. org/30730/

2. Consórcio Brasileiro de Acreditação de Sistemas e Serviços de Saúde. Padrões de acreditação da Joint Comission International para hospitais. $3^{\text {a }}$ ed. Rio de Janeiro: CBA; 2008.

3. World Health Organization (WHO). Summary of the evidence on patient safety: implications for research. Edição: Ashish Jha; 2008.

4. Kohn LT, Corrigan JM, Donaldson MS. To err is human: building a safer health system. Washington (DC): National Academy Press; 2001.

5. Gallotti RMDG. Eventos adversos - o que são? Rev. Assoc. Med. Bras. 2004;(50):109-26.

6. Ministério da Saúde (BR). Agência Nacional de Vigilância Sanitária (ANVISA). Boletim Informativo Segurança do Paciente e Qualidade em Serviços de Saúde [Internet]. Brasília; 2011 [acesso em 20 jul 2013]. Disponível: http://portal.anvisa.gov.br/wps/ wcm/connect/f72c20804863a1d88cc88d2bd5b3ccf0/ BOLETIM+I.PDF?MOD=AJPERES 
7. Bork AMT. Segurança do paciente: a enfermagem como agente de mudança [editorial]. Einstein. 2007;5(Suppl11).

8. Vargas MAO, Luz AMH. Práticas seguras do/no cuidado de enfermagem no contexto hospitalar: é preciso pensar sobre isso e aquilo. Enferm. Foco. 2010;1(1):23-7.

9. Silva AEBC. Segurança do paciente: desafios para a prática e a investigação em Enfermagem [editorial]. Rev. Eletr. Enf. [Internet]. 2010;12(3):422 [acesso em 01 ago 2013]. Disponível: http://revistas.ufg.br/index. php/fen/article/view/11885/7815

10. Killam LA, Phyllis M, Raymond JM, Mossey S, Timmermans KE, Binette J. Unsafe clinical practices as perceived by final year baccalaureate nursing students: Q methodology. BMC Nursing. 2012;11:26.

11. Leung GK, Patil NG. Patient safety in the undergraduate curriculum: medical students' perception. HKMJ. 2010;16(2):101-5.

12. Wong BM, Etchells EE, Kuper A, Levinson W, Shojania KG. Teaching quality improvement and patient safety to trainees: A systematic review. Acad. Med. 2010;85(9):1425-39.

13. Yoshikawa JM, Sousa BEC de, Peterlini MAS, Kusahara DM, Pedreira MLG, Avelar AFM. Compreensão de alunos de cursos de graduação em enfermagem e medicina sobre segurança do paciente. Acta Paul. Enferm.2013;26(1):21-9.

14. Ministério da Saúde (BR). Portaria n. 1.377 de 9 de Julho de 2013. Aprova os Protocolos de Segurança do Paciente [Internet] 2011 [acesso em 13 fev 2014]. Brasília; 2013 Disponível: http://bvsms.saude.gov.br/ bvs/saudelegis/gm/2013/prt1377_09_07_2013.html

15. Ministério da Saúde (BR). Portaria n. 2.095 de 24 de Setembro de 2013. Aprova os Protocolos Básicos de Segurança do Paciente. Brasília; 2013 [acesso em 13 fev 2014]. Disponível: http://bvsms.saude.gov.br/bvs/ saudelegis/gm/2013/prt2095_24_09_2013.html

16. Polit DF, Beck CT. Nursing research: principles and methods. $7^{\mathrm{a}}$ ed. Philadelphia (PA): Lippincott Williams \& Wilkins; 2004.

17. Zanon U. Qualidade da assistência médico-hospitalar. Conceito, avaliação e discussão dos indicadores de qualidade. Rio de Janeiro (RJ): Medsi; 2001.

18. Adami NP, Cunha ICKO, D’Innocenzo M. O movimento pela qualidade nos serviços de saúde e enfermagem. Rev. bras. enferm. 2006;59(1):84-8.

19. Laus AM, Anselmi ML. Caracterização dos pacientes internados nas unidades médicas e cirúrgicas do HCFMRP-USP, segundo o grau de dependência em relação ao cuidado de enfermagem. Rev. Latino-Am. Enfermagem. 2004;12(4):643-9.

20. Martínez QAA, Hueso MC, Gálvez GG. Fortalezas e ameaças em torno da segurança do paciente segundo a opinião dos profissionais de enfermagem. Rev. LatinoAm. Enfermagem. 2010;18(3):8.

21. Berquó L. Serviço de epidemiologia e gestão de risco. Porto Alegre: Hospital Mãe de Deus; 2007.

22. Paiva MCMS, Paiva SAR, Berti HW. Eventos adversos: análise de um instrumento de notificação utilizado no gerenciamento de enfermagem. Rev Esc Enferm USP. 2010;44(2):287-94

23. Praxedes MFS, Telles Filho PCP. Identificação de erros no preparo e administração de medicamentos pela equipe de enfermagem e das ações praticadas pela instituição hospitalar. Cogitare enferm. 2008;13(4):514-9.

24. Cassiani SHB, Freire CC, Gimenes FRE. A prescrição médica eletrônica em um hospital universitário: falhas de redação e opiniões de usuários. Rev Esc Enferm USP. 2003;37(4):51-60.

25. Paiva SMA. Qualidade da assistência hospitalar: avaliação da satisfação dos usuários durante seu período de internação [tese]. Ribeirão Preto: Escola de Enfermagem de Ribeirão Preto, Universidade de São Paulo; 2006. p.166

26. Kuwabara CCT, Évora YDM, Oliveira MMB. Gerenciamento de risco em tecnovigilância: construção e validação de instrumento de avaliação de produto médico-hospitalar. Rev. Latino-Am. Enfermagem. 2010;18(5):9.

27. Carraro TE. Humanização: subsídios para a metodologia da assistência de enfermagem. In: Westphalen MEA, Carraro TE. Metodologia para a assistência de enfermagem: teorizações, modelos e subsídios para a prática. Goiânia: AB; 2001.

28. Ghandi TK, Seger DL, Bates DW. Identifying drug safety issues: from research to practive. Int. J. Qual. Health Care. 2000;12:69-76.

29. Berlitz FA. Controle da qualidade no laboratório clínico: alinhando melhoria de processos, confiabilidade e segurança do paciente. J. bras. patol. med. lab. 2010;46(5):353-63. 